\title{
Measuring the Attitudes of Patients Towards Diabetes and its Treatment
}

\author{
Robert M. Anderson, Michael B. Donnelly and Robert F. Dedrick \\ The University of Michigan Medical School, Michigan Diabetes Research and Training Center, Department of Postgraduate \\ Medicine/Health Professions Education, Ann Arbor, MI 48109-0201 (U.S.A.)
}

(Received January 5th, 1990)

(Accepted June 8th, 1990)

\begin{abstract}
The following is a study of the general diabetes-related attitudes of 1202 patients with diabetes in Michigan. The instrument used to measure attitudes was a revised version of the Diabetes Attitude Scale (DAS) designed to measure the attitudes of health care professionals. A factor analysis of the revised DAS yielded seven diabetes attitude factors. These factors represented patients' attitude toward: (1) the need for special training in order to provide diabetes care; (2) patient compliance; (3) the seriousness of noninsulin-dependent diabetes (NIDDM); (4) the relationship between blood glucose levels and complications; (5) the impact of diabetes on the patient's life; (6) patient autonomy; (7) team care. Overall, the respondents to this survey displayed attitudes consistent with the current recommendations of health care professionals in the field of diabetes. The revised DAS is an instrument that can be used to measure and compare the attitudes of both health care professionals and patients on a variety of diabetes-related issues.
\end{abstract}

R.M. Anderson, EdD is Assistant Professor.

M.B. Donnelly, PhD is Associate Professor.

R.F. Dedrick, $\mathrm{PhD}$ is Assistant Professor.
Keywords: Diabetes; Attitudes; Patients; Health care professionals.

\section{Introduction}

\section{Background}

In 1975 the National Diabetes Commission issued its report to Congress [1]. In that report the Commission suggested that the diabetes-related attitudes of health care professionals (HCPs) were often inappropriate and that these attitudes could lead to negative outcomes for patients. The Commission did not present scientific evidence to support its claims which appeared to be based on personal experience and anecdotal evidence. In order to determine if the assertions of the Commission would be supported by research, the authors, with the help of a national panel of experts in diabetes, developed the Diabetes Attitudes Scale (DAS) for health care professionals [2].

The content of the DAS was developed through the efforts of a national panel of 17 diabetes experts. The panel included 3 physicians, 3 nurses, 4 nutritionists, 3 consumers of diabetes care and 4 behavioral scientists. The group interacted by mail through a modified Delphi [3] process. Members were asked 
to write Likert type attitude items, i.e., statements that are responded to on a five-point scale that indicates the respondents' degree of agreement or disagreement with the statements. To ensure the comprehensiveness of the DAS, the panel was asked to write items covering four global areas of diabetes: the disease itself, treating diabetes, diabetic patients and professional education in diabetes. The panel wrote a total of 347 attitude items: 62 items related to the disease itself, 135 items related to treating diabetes, 92 items focused on the individuals with diabetes and 58 items on diabetes professional education.

The panel members reviewed all 347 items, suggested wording changes, indicated whether the correct response to an item was to agree or disagree and selected 20 items from each of the 4 areas that they believed addressed important issues in diabetes and therefore should be included in the final scale. The group was asked to indicate the correct response for each item to provide a criterion for desirable or appropriate attitudes. This criterion was established because one of the intended uses of the scale was the evaluation of professional education programs. Items that had at least an $80 \%$ level of agreement on the direction of the appropriate response and more than five votes for inclusion were included in a preliminary version of the scale. This resulted in an instrument for which there was a high level of agreement among the panel of diabetes experts regarding both the significance of the items and their correct responses. The preliminary version of the scale which contained 60 items was pilot tested using a convenience sample of 60 HCPs. An item analysis, examining item variability and inter-item correlations, was performed and led to the elimination of 10 items.

Psychometric analyses of the 50 items using a sample of 633 nurses, 322 dietitians and 116 physicians identified eight factors. These factors represented HCPs' attitudes toward: (1) the need for special training in the treatment of diabetes; (2) the importance of blood glucose control in minimizing the complications of diabetes; (3) the role of the patient in diabetes self-care and management; (4) patients' commitment to controlling their disease; (5) the importance of a team approach to diabetes care; (6) the seriousness of noninsulin-dependent diabetes (NIDDM); (7) the difficulties in treating diabetes; (8) the efficacy of outpatient education. Information about the reliability and validity of the DAS has been published elsewhere [4].

In reviewing the responses to the DAS by physicians, nurses and dietitians, it became clear that it would be useful to know how persons with diabetes view these same issues. Additionally, a DAS that could be completed by both patients and health care professionals would allow for direct comparisons of the diabetes-related attitudes of both groups. In assessing the potential of the DAS to measure the attitudes of diabetic patients, it was decided that the wording of the instrument was too technical for patients and would nced to be changed. The authors rewrote most of the items to make them less technical while trying to retain the original meaning of the items. A study [5] was carried out in which two random samples of health care professionals were sent either the original DAS or the revised DAS. A comparison of the responses of health care professionals to the original DAS and the revised DAS clearly indicated that the revisions had changed the psychometric properties of the attitude scale. Based on these results it was determined that the revised DAS would have to be viewed as a new attitude measure and its psychometric properties would have to be established based on administrations of the scale to patients.

This study focuses on the responses to the revised DAS by diabetic patients in Michigan. This paper presents the psychometric properties of the revised DAS, a description of the diabetes-related attitudes of the patients and a comparison of the patients' attitudes to their 
demographic characteristics and diabctes history. It is important to note that the revised DAS focuses on issues of concern to both patients and health care professionals. This instrument is not intended to be, or to replace, a diabetes attitude scale focusing exclusively on the concerns of persons with diabetes. Scales focusing exclusively on the concerns of patients have been developed to measure such constructs as Locus of Control [6] and a variety of health beliefs derived from the Health Belief Model [7-10]. These scales have been used mainly to identify facilitators and barriers to patient adherence to treatment regimens.

The present DAS represents a more generalized measure of patients' attitudes toward diabetes and is designed to facilitate comparisons between patients and health care professionals. A comparison of the attitudes of HCPs and patients provides an opportunity to identify differences in opinion that could interfere with the quality of the patient-HCP relationship and ultimately affect the management and treatment of the disease. The present DAS can also be used to assess the impact of diabetes education programs and on the attitudes of patients and to explore the relationship between attitudes and behavior .

\section{Theoretical orientation}

The attitude-behavior theory of Fishbein and Ajzen was used in the present study to understand and describe the diabetes-related attitudes and their potential relationship to the behavior of patients. Reasoned action was chosen because it provides a useful, theoretical model and because it is an empirically validated attitude behavior model that allows the integration of behavior/attitude theories previously applied to diabetes such as the health belief model and the concept of self-efficacy [11]. Reasoned action also allows for the inclusion of variables such as social environment, demographics and personality traits into a theory of diabetes-related behavior. Fishbein and Ajzen's theory [12] of reasoned action posits that the best predictor of a patient's behavior is that patient's intention to behave in a certain way. For example, if one were trying to determine the likelihood that a patient would engage in an intensive treatment regimen in order to obtain tight blood glucose control the theory of reasoned action indicates that the best predictor of whether a patient will in fact carry out that regimen is a patient's intention to do so.

Fishbein and Ajzen's theory states that a patient's intention to behave in a certain way has two major determinants. The first is the subjective norm, i.e., whether other people whom the patient views as important feel positively or negatively about trying to achieve tight glucose control. The second determinant is the patient's attitude toward the behavior, i.e., how positively or negatively the patient feels about trying to achieve tight glucose control. The patient's attitude will be a summation of that patient's specific beliefs about the behavior. For example, does the patient believe that tight glucose control is achievable? Does the patient believe that tight glucose control will prevent complications?

In the study that follows, the summary statements describing the seven factors should be considered as attitudes in the FishbeinAjzen model and the individual items that comprise each of the seven factor subscales should be understood as salient belicfs underlying those attitudes.

\section{Materials and methods}

\section{The revised Diabetes Attitude Scale}

The revised DAS consists of 50 Likert scale items (5, strongly agree; 4 , agree; 3 , neither agree or disagree; 2 , disagree; 1 , strongly disagree). The DAS was revised in order to simplify the wording of the original items designed for HCPs. Forty-one of the original 50 items were rewritten to eliminate technical terms. Although the revisions were not specifically intended to lower the reading level of 
the DAS, the rewording lowered the reading level from 12 th grade to 10 th grade. The content of the items on the revised DAS very closely matches the original items and 9 of the items from the original DAS were not changed. However, as noted earlier, in this paper, the meaning of many of the items changed as a result of these modifications. The revised DAS questionnaire also contains questions regarding general and diabetes-specific demographic measures and diabetes history.

\section{Study participants}

Surveys were mailed to two samples of diabetic patients. The first sample consisted of 1054 patients who had attended the University of Michigan Hospitals' diabetes clinic. The survey was returned by 419 patients in this sample for a return rate of $40 \%$. To broaden the sample of patients, the survey was also sent to 1003 patients in nine Michigan communities who were receiving a monthly diabetes newsletter. Patients in this sample returned 823 surveys for a return rate of $82 \%$. In total, 2057 surveys were sent to patients and 1242 surveys were returned for an overall return rate of $60 \%$. Forty surveys were subsequently eliminated from the analyses because the patients did not meet the age criterion (age $\geqslant 16$ years). The final sample consisted of 1202 patients.

The demographic characteristics of this sample of 1202 patients with diabetes are summarized in column 2 of Table IV. One point of interest in this table is the comparison of the self-reported type of diabetes versus the calculated classification (e.g., NIDDM diabetes $66 \%$ calculated vs. $45 \%$ reported). The questionnaire asked patients to indicate whether they had insulin-dependent (IDDM) or noninsulin-dependent diabetes (NIDDM). The questionnaire contained a short explanation of each type of diabetes and included the fact that many patients with NIDDM diabetes take insulin. However, in previous surveys of diabetic patients it was learned that a significant number of patients do not know which type of diabetes they have and a significant number of patients with NIDDM who take insulin assume they have IDDM. Therefore, it was necessary to apply a formula developed by Davis et al. [13] to correctly classify the patients. The Davis formula uses age of onset, insulin use and percent of ideal body weight to classify patients. It has been found to be $93 \%$ accurate when compared to the stimulated C-peptide test for classifying the type of diabetes. Table IV contains both the self-reported type of diabetes and the type of diabetes as determined by the formula.

\section{Statistical methods}

Since a major purpose of this study was to determine the psychometric properties of the revised DAS, a variety of statistical analyses were conducted. To determine if there were empirically derivable subscales, a principal axes factor analysis was performed. The scree test [14] was used to determine the number of common factors and the factor structure was rotated using both varimax (uncorrelated factors) and oblimin (correlated factors) rotation methods. Both methods of rotation were used since it was not known a priori which would better represent the inter-item correlations. Factor loadings (the correlations of items with the factors) of 0.30 or greater were considered educationally significant (note that because of the large sample size, factor loadings of small magnitude would be statistically significant) and were used to define the factors. Cronbach's $\alpha$, the standard error of measurement (the estimated standard deviation of an individual's score if the scale were administered many times) and related item statistics were calculated for each of the factor subscales. A score was calculated for each subscale by averaging the items which defined the subscale. These subscale scores were then intercorrelated using Pearson productmoment correlations.

On each of the subscales, individuals were 
classified into one of two groups. If an individual's score on a given subscale was less than or equal to 3.00 (the subscale midpoint), he/she was placed in the negative attitude group ( $\mathrm{Neg}$ ). If an individual's score was greater than 3.00 , he/she was placed in the positive attitude group (Pos). Differences between these two groups on a variety of demographic and self-care measures were determined by means of independent groups $t$-tests. A multivariate analysis of variance was performed on each factor using all the demographic measures to ensure that overall there were significant group differences. Because of the large sample size, the more conservative 0.01 level of significance was chosen to define a significant difference.

\section{Results}

A principal axes factor analysis of the 50 DAS items showed that there were 13 eigenvalues greater than 1.0. An examination of the scree plot [14] of the 50 eigenvalues suggested that an eight-factor solution would adequately represent the data. According to this criterion the number of factors is taken as the first number that does not fall on the straight line (drawn from right to left). This eight-factor solution was then rotated using both the varimax criterion and the oblimin criterion. A comparison of these two solutions indicated that the varimax more closely approximated simple structure in that fewer items loaded significantly ( 0.30 or greater) on more than one factor with the varimax than with the oblimin solutions.

Measures of internal consistency (Cronbach's $\alpha$ ) were calculated for the items comprising the eight factors. All of the factors possessed good internal consistency (alphas $>$ 0.60) except for Factor 8 which contained three items and had an alpha of 0.37. This factor was dropped from further analyses. For the remaining seven factors, items that were not easily interpreted in view of the other items defining the factor and whose elimination did not markedly decrease the reliability were deleted at this time. This process resulted in the elimination of nine items. The final DAS consisted of 34 items and seven factors. To ensure that the item deletions had not modified the fundamental factor structure, the 34 items were factor analyzed and rotated (varimax) to a sevenfactor solution.

The structure was replicated and it was found that this solution accounted for $34 \%$ of the total variance. Factor 1 accounted for $12.9 \%$ of the variance (eigenvalue $=4.4$ ); Factor 2 explained $7.4 \%$ (eigenvalue $=2.5$ ); Factor 3 explained $1.7 \%$ (eigenvalue $=0.6$ ); Factor 4 explained $2.0 \%$ (eigenvalue $=0.7$ ); Factor 5 explained $4.3 \%$ (eigenvalue $=1.5$ ); Factor 6 explained $2.4 \%$ (eigenvalue $=0.8$ ); Factor 7 explained $3.5 \%$ of the variance (eigenvalue $=1.2$ ). Table $I$ contains the items defining each factor along with their varimax factor loadings for the factor on which the item loads most highly. Structure of the revised DAS is very similar to the original DAS. The instruments share six common factors. The mean difference between the alphas of the six common factors is $\mathbf{0 . 0 7}$. Item statistics are also included in Table I.

Factor 1 was labeled Special Training and indicates the extent to which respondents believe that health care professionals need special training to care for persons with diabetes. Factor 2 was called Patient Compliance and indicates the extent to which respondents support the idea that patients should do what they are told to do by health care professionals. There is a moralistic (i.e., blaming the patient) tone to a number of the items in this subscale. Factor 3 was called Seriousness of NIDDM and indicates the extent to which the respondents view NIDDM as a serious disease. Factor 4 was called Relationship Between Blood Glucose Levels and Complications and indicates the extent to which the respondents perceive a relationship between blood glucose levels and the onset of the 
Table I. Factor analysis and item analysis of the revised diabetes attitude scale.

$\begin{array}{lll}\begin{array}{l}\text { Factor } \\ \text { loadings }\end{array} & \text { Mean } \pm S D & \begin{array}{l}\text { Corrected } \\ \text { item/scale } \\ \text { correlation }\end{array}\end{array}$

\section{Factor 1: Need for Special Training}

In general, I believe that:

21 ... health care professionals who treat people with diabetes should be trained to communicate well with their patients

$12 \ldots$... it is important for the nurses and dietitians who teach people to care for their diabetes to learn counseling skills

13 ... health care professionals should be required to continue to learn about diabetes because diabetes care is changing fast

1 ... health care professionals need to have special training to provide effective treatment of diabetes

38 ... nurses and dietitians who have special training in diabetes will give better care to patients

45 ... diabetes education for health care professionals should cover diabetes in the elderly

42 ... to do a good job, diabetes educators should learn a lot about being teachers

\section{Factor 2: Attitude Towards Patient Compliance}

In general, I believe that:

28 . . people who do not follow their recommended diabetes treatment don't really care about controlling their diabetes

4 ... controlling their diabetes should be the most important thing in the lives of people with diabetes

41 ...the parents of diabetic teenagers should be in charge of how their children take care of their diabetes

43 ... decisions about caring for diabetes should be made by the doctor

32 ...telling patients about the complications of diabetes will scare them into following their recommended treatment

$24 \quad \ldots$ if people with diabetes do not cooperate and follow their recommended treatment there is not much that health care professionals can do for them

$\begin{array}{llll}0.55 & 4.50 & 0.55 & 0.49 \\ 0.59 & 4.24 & 0.68 & 0.51 \\ 0.50 & 4.48 & 0.61 & 0.47 \\ 0.37 & & & \\ 0.38 & 4.63 & 0.59 & 0.37 \\ & & & \\ 0.45 & 4.24 & 0.66 & 0.41 \\ 0.41 & 4.18 & 0.65 & 0.41 \\ & & & 0.33\end{array}$

0.61

1.17

0.48

0.49

4.25

0.90

0.36

0.52

1.16

0.43

0.47

3.52

0.99

0.43

0.41

2.91

1.08

0.34

0.43

4.13

0.89

0.34 
Table I (continued)

$\begin{array}{lll}\begin{array}{l}\text { Factor } \\ \text { loadings }\end{array} & \text { Mean } \pm \text { SD } & \begin{array}{l}\text { Corrected } \\ \text { item } / \text { scale } \\ \text { correlation }\end{array}\end{array}$

\section{Factor 3: Seriousness of Noninsulin-Dependent}

In general, I believe that:

10 ... noninsulin-dependent diabetes is a

less serious disease than insulin-dependent diabetes ${ }^{a}$

18 . . . people whose diabetes is treated by

0.90

0.38 just a diet do not have to worry about getting many long-term complications of diabetes ${ }^{a}$

$6 \quad$... diabetes that can be controlled by just being on a diet is a pretty mild disease $^{a}$

\section{Factor 4: Blood Glucose Control and Complications}

In general, I believe that:

26 . . good blood sugar control will reduce

14 ... people with diabetes who have poor have diabetes complications than people who have a good blood sugar control

49 ... having high blood sugar over a long period of time is linked to getting longterm diabetic complications

35 ... there is not much use in trying to have good blood sugar control because the complications of diabetes will happen anyway ${ }^{\mathrm{a}}$

\section{Factor 5: Impact of Diabetes On Patients' Lives}

In general, I believe that:

22 ... diabetes affects almost every part of a diabetic person's life pretty smalla 
Table I (continued)

\begin{tabular}{lll}
$\begin{array}{l}\text { Factor } \\
\text { loadings }\end{array}$ & Mean $\pm S D$ & $\begin{array}{l}\text { Corrected } \\
\text { item/scale } \\
\text { correlation }\end{array}$ \\
\hline
\end{tabular}

Factor 6: Attitude Towards Patient Autonomy

In general, $I$ believe that:

46 ...the important decisions regarding daily diabetes care should be made by the person with diabetes

11 ... people with diabetes should choose their own goals for diabetes treatment

48 ... people with diabetes should learn a lot about the disease so they can be in charge of their own diabetes care

3 ... people with diabetes should be taught how to choose their own self-care methods (e.g., type of diet, type of blood sugar monitoring, number of daily insulin injections)

37 ... people with diabetes have the right to decide how hard they will work to control their blood sugar

\section{Factor 7: Attitudes Towards Team Care}

In general, I believe that:

34 ... doctors do not need help from

$4.24^{\mathrm{a}}$

0.71

0.45 nurses and dietitians to treat patients with diabetes ${ }^{\mathrm{a}}$ about diabetes care to patients, physitians

29 ... doctors should send people with diabetes to a dietitian to help them with their diet

47 ... doctors should send people with diabetes to a nurse educator to help them learn about their diabetes

Reverse scales.

complications of diabetes. Factor 5 was called Impact of Diabetes on the Patient's Life and indicates the extent to which respondents believe that diabetes has a significant negative impact on the patient's life. Factor 6, Patient Autonomy indicates the extent to which respondents agree that the patient should be the primary decision maker regarding the daily self-care of diabetes. Factor 7, Team Care, indicates the extent to which respondents are supportive of the need for nurses and dietitians in the care of diabetes. 


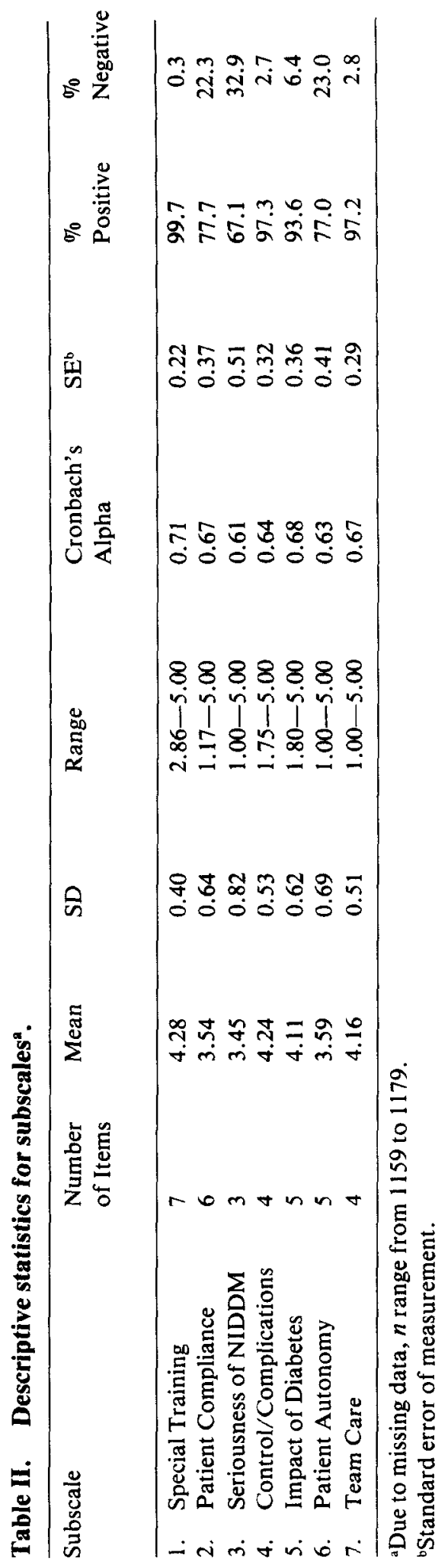


Table II presents descriptive statistics and Cronbach $\alpha s$ for each of the factorially defined subscales. The percentage of respondents who reported positive $(>3.0)$ or negative $(\leqslant 3.0)$ attitudes for each of the seven subscales is presented in the last two columns of Table II. In general, the majority of the respondents strongly believed that: HCPs need special training to care for persons with diabetes; good blood glucose control reduces the likelihood that complications will develop; diabetes has a significant negative impact on the patient's life; that a team approach is essential to diabetes care. Although the respondents generally agreed that NIDDM is a serious disease and were supportive of patients being in charge of their diabetes management, these issues generated the widest differences in opinion among the respondents.

Table II also presents the reliabilities (a) and related standard errors of measurement for the seven subscales. The reliabilities ranged from 0.61 for the scriousncss of NIDDM ( 3 items) to 0.71 for the need for special training ( 7 items). These reliabilities were viewed as adequate for making group comparisons.

Pearson product-moment correlations for the seven subscales are presented in Table III. The strongest correlation between the subscales was between the nced for special train- ing and the need for a team approach to diabetes care $(r=0.50)$ The remaining correlations were low to moderate indicating that the subscales were measuring relatively independent attitudes, although it is recognized that the subscale reliabilities are attenuating these correlations to some degree.

Table IV presents the means for the positive attitude groups (Pos) and the negative attitude groups (Neg) for selected subscales on a variety of demographic and diabetes specific measures. In the Fishbein-Ajzen model demographics are considered external variables. External variables may influence the beliefs a patient has or the relative importance that patients attach to attitudinal and normative considerations [11]. Three of the subscales (Special Training, Control/ Complications, Team Care) were not included since the vast majority of the sample had favorable attitudes and the size of the Neg group contained less than $5 \%$ of total sample (see Table II). The equality of each pair of means was tested by independent groups $t$-tests. In addition, a multivariate analysis of variance was carried out for each subscale to ensure that there were overall differences. For each subscale the multivariate $F$ was statistically significant $(P<0.01)$.

Inspection of Table IV indicates that gender, age, understanding of diabetes and type of diabetes frequently differentiated positive

Table III. Pearson product-moment correlations between subscales.

\begin{tabular}{|c|c|c|c|c|c|c|c|c|}
\hline & & 1 & 2 & 3 & 4 & 5 & 6 & 7 \\
\hline 1. & Special Training & - & & & & & & \\
\hline 2. & Patient Compliance & 0.11 & - & & & & & \\
\hline 3. & Seriousness of NIDDM & 0.07 & -0.19 & - & & & & \\
\hline 4. & Control/Complications & 0.35 & 0.10 & 0.09 & - & & & \\
\hline 5. & Impact of Diabetes & 0.35 & -0.11 & 0.10 & 0.16 & - & & \\
\hline 6. & Patient Autonomy & 0.21 & -0.24 & -0.05 & 0.09 & 0.19 & - & \\
\hline & Team Care & 0.50 & 0.12 & 0.12 & 0.38 & 0.24 & 0.09 & - \\
\hline
\end{tabular}




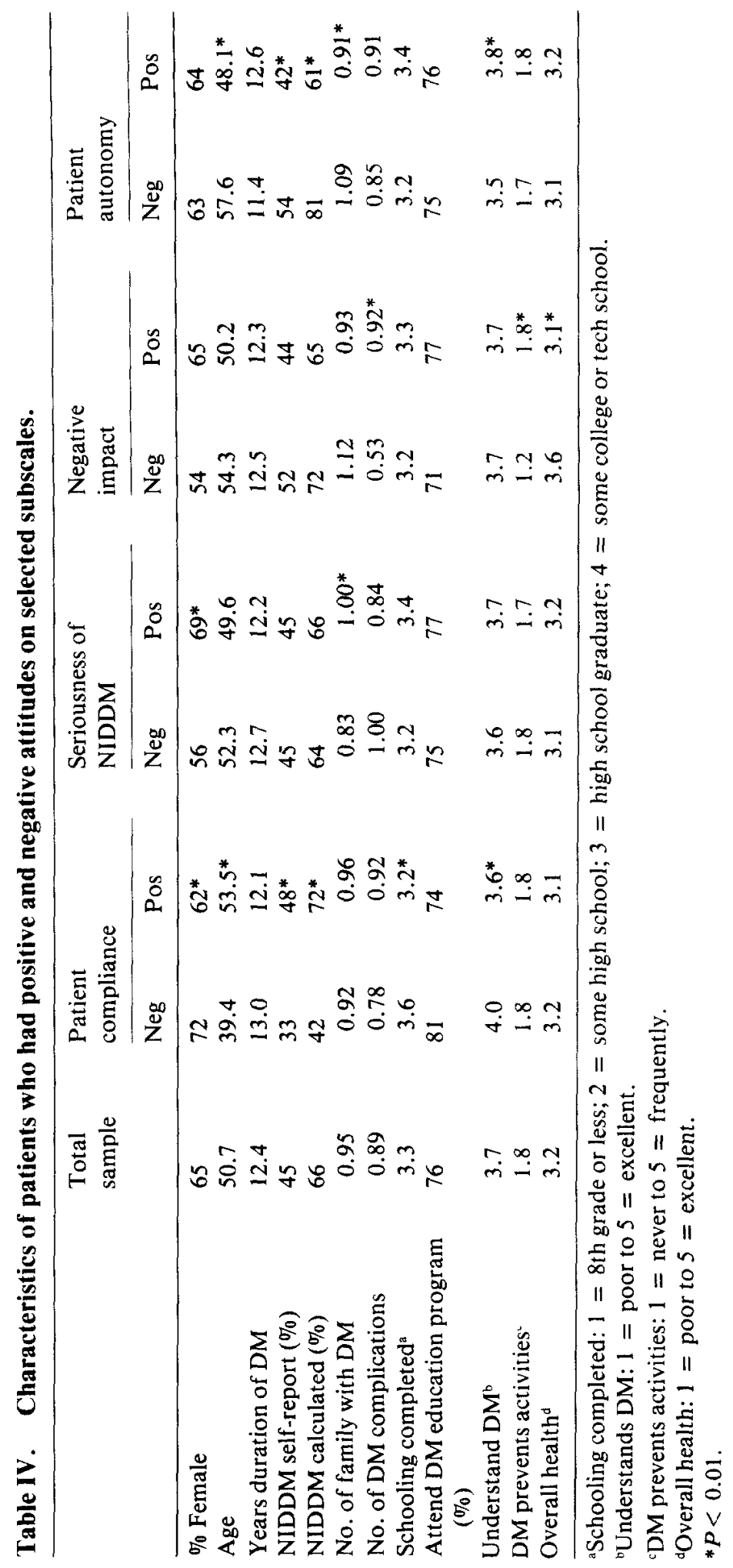


and negative groups. In contrast, duration of diabetes and having attended an education program did not affect the attitudes measured in this study. Attitudes toward patient compliance and patient autonomy were most influenced by the demographic and diabetes care measures, while the attitude toward the seriousness of NIDDM was influenced the least.

\section{Discussion}

\section{Psychometric properties of revised DAS}

The use of a panel of diabetes experts to develop the items and the psychometric analyses conducted on the revised DAS provide preliminary support for its validity and reliability as a general measure of the diabetesrelated attitudes of patients. In using the revised DAS it should be noted that the subscales are reliable enough to compare the attitudes of groups of diabetic patients. However, the reliabilities are low enough that if the subscales are used to compare the score of a single patient at two different time points the standard error of measurement must be explicitly taken into account. Further studies will have to be conducted to gather additional evidence regarding the construct validity of the revised DAS.

\section{Revised DAS factors}

Factor 1: attitude towards the need for special training. Overall the respondents to the revised DAS agreed most strongly with this set of items indicating the need for health care professionals to have special training to care for diabetic patients. In fact the need for special training was the only attitude on which virtually all the respondents agreed. This is not a surprising finding since one would expect patients to believe that the health care professionals who treat them should have as much training as possible to provide that treatment.

Factor 2: attitude towards patients compliance. This is an interesting factor because it is not only a composite of items that are related conceptually but represents items that are related by their moralistic or blaming tone as well. Overall, the patients in our survey agreed that patients should do what they are told, though the strength of the agreement was significantly weaker than with Factor 1. Also, a significant minority of patients disagreed with these items. There were a number of interesting differences between the patients who felt patients should do what they are told to do and those patients who did not feel this way. The patients who disagreed were more likely to be female, younger and have IDDM (both actual and perceived). Also, the patients who disagreed rated their understanding of diabetes higher and had completed more schooling.

Factor 3: attitude toward the seriousness of NIDDM. Overall, the majority of the respondents viewed NIDDM as a serious disease. The patients who were most likely to feel that NIDDM was a serious disease tended to be female and have more family members who had diabetes. It is important to remember that for many patients the type of diabetes that they believe they have is determined by whether they take insulin or not. For these patients taking insulin is a message about how serious this disease is.

Factor 4: attitude towards the relationship between blood glucose levels and complications. Almost the entire sample agreed that there was a relationship between elevated levels of blood glucose and the onset of the complications of diabetes. This finding is not surprising in light of the fact that most diabetic patients are encouraged to control their blood glucose in order to delay, prevent, or minimize the complications of diabetes.

Factor 5: attitude towards the negative impact of diabetes on the patient's life. This subscale measured the extent to which patients thought that diabetes had a negative impact on the quality of patients' lives. The great majority of the respondents agreed that 
1. Attitude towards the need for special training

2. Attitudes toward patient compliance

3. Attitude towards the seriousness of noninsulin-dependent diabetes (NIDDM)

4. Attitude towards the relationship of blood glucose levels and complication

5. Attitude towards the negative impact of diabetes on the patient's life

6. Attitude towards patient autonomy

7. Attitude towards team care
The strong agreement by the respondents with the need for special diabetes training provides support for the concept of specializing in diabetes care and education; the finding that patients desire special expertise on the part of health care providers is consistent with the movement in the diabetes community toward the recognition of patient education programs which meet national standards and the certification of diabetes educators

This factor indicates that a significant number of patients do not want to be "told" what they should do to care for their diabetes; this especially applies to younger, better educated patients with IDDM; the more schooling that patients have completed and the more they perceive themselves to understand diabetes the more likely they are to resist being told what to do; it is important to determine the patient's need for structure and/or flexibility in the self-care plan and respond appropriately

It is important to strike a balance between false reassurance and unnecessary fear when discussing noninsulin-dependent diabetes (NIDDM) with patients; many patients have been told that they have a "touch of diabetes" or "mild diabetes" which misleads them into believing that diabetes is not a serious disease; such false reassurance makes it very difficult for diabetes educators to facilitate important and difficult lifestyle changes required for the treatment of NIDDM; Patients need to understand that diabetes is a serious disease and that their role in its self-care is very important

The fact that most patients believe in the relationship between blood glucose control and complications can be viewed as a positive finding; but educators must be careful not to implicitly or explicitly guarantee patients that they will escape the complications of diabetes if they engage in an aggressive effort to control their blood glucose levels; it is also important for educators to help patients minimize guilt and self-blame if they do develop the complications of diabetes

The small minority of patients who claim diabetes did not interfere with the quality of their life tended to view themselves as healthier than the majority of patients who asserted that diabetes did have a negative impact on the quality of their lives; this finding suggests that diabetes and its complications detracts significantly from the quality of life for most patients; diabetes educators should avoid suggesting that people with diabetes can lead a "normal life," an assertion which many patients resent

The finding that older patients are more likely to want their physician to be in charge of their diabetes care has important clinical implications; although a great many diabetes educators value patient autonomy and see it as one of the major outcomes of diabetes education, it is important to remember that a significant number of patients do not desire an independent self-care role

The great majority of patients value team care; this finding has similar implications to the findings on Factor 1, i.e., patients view diabetes as a serious and complex disease and value a comprehensive, multidisciplinary approach to care 
diabetes detracted from the quality of patients' lives. The small minority of patients who disagreed with this view reported a better degree of overall health, a lower number of diabetes-related complications and indicated that diabetes rarely prevented them from performing important daily activities.

Factor 6: attitude towards patient autonomy. The items comprising this factor asked patients to chose between whether the physician or the patient should be the primary decision-maker in the daily treatment of diabetes. Overall, the respondents agreed that the patient should be the primary decisionmaker. However, the variance of the scores around this attitude was the largest of all of the factors, indicating that significant differences of opinion about this issue existed among the respondents. The patients who were supportive of patient autonomy tended to be younger and were more likely to have IDDM. In addition, these patients were likely to have fewer family members with diabetes and report a better understanding of diabetcs.

Factor 7: attitude towards team care. This factor measured the extent to which the respondents viewed nurses and dietitians as having an important role in the treatment of diabetes. Respondents were generally supportive of the role played by nurses and dietitians, with only a very small minority of patients believing that team care was not essential for the treatment of diabetes.

The present findings indicate that the majority of patients believe that HCPs need special training to care for patients with diabetes and that a multidisciplinary team approach is essential to providing effective treatment for diabetes. These beliefs suggest that patients view diabetes as a serious, complex and multidimensional disease. Patients, however, generally believe that they have some control over the outcomes of the disease. In general, most patients believe that maintaining good blood glucose control will delay, prevent, or minimize the complications of diabetes.

The issue of patient autonomy in diabetes management generated a wide range of opinion in this study, with younger patients preferring more autonomy in the daily management of diabetes than older patients. In general, older patients believed that the doctor should be the primary decision-maker in the daily treatment of diabetes and that patients should do what they are told to do. Although this belief is consistent with the traditional view of the doctor-patient relationship, the belief may be counterproductive in the management of a chronic disease like diabetes which requires daily self-management behaviors (e.g., self-monitoring of blood glucose) and frequent decisions about medication, diet and activity levels.

Differences in attitudes among patients with diabetes will affect how they receive and act on the information provided to them during education and counseling sessions. Based on our clinical and research experience in diabetes education we have compiled Table $\mathrm{V}$ that suggests some implications for practice for some of the major variations and attitudes found among this sample of patients.

\section{Summary}

The revised DAS is an instrument that can be used to measure a variety of relatively independent attitudes of both health care professionals and patients. The reliabilities of its subscales, although modest, are adequate for group comparisons. The revised DAS can be used to compare the attitudes of health care professionals with those of patients which could indicate differences that should be the focus of diabetes patient education. The revised DAS can also be used to assess the impact of diabetes education on the attitudes of both groups. The revised DAS can also be used to describe the relationship between the attitudes of both patients and health care professionals and their diabetesrelated behavior.

\section{Acknowledgment}

This study was supported in part by a grant from the Diabetes Research and Education Foundation and by National Insti- 
tutes of Health Grant No. 3P60AM20572, National Institute of Diabetes, Digestive and Kidney Diseases.

\section{References}

1 Report of the National Commission on Diabetes to the Congress of the United States, Vol. 3, Part 5. Washington, DC: US Department of Health, Education and Welfare, 1975, p. 5-13 (NIH Publ. No. 76-1024).

2 Anderson RM, Gressard C: Developing a measure of the attitudes of health care providers toward diabetes and its treatment. Diabetes 1987; 36: 120A.

3 Udinsky BF, Ostorlind SJ, Lynch S: Delphi technique. In: Evaluation Resource Handbook: Gathering, Analyzing, Reporting Data. San Diego, CA: Edits, 1981.

4 Anderson RM, Donnelly MB, Gressard C, Dedrick RF: The development of a diabetes attitude scale for health care professionals. Diabetes Care 1989; 12: 120-127.

5 Anderson RM, Donnelly MB: Words and meaning: a cautionary tale for diabetes educators. Diabetes Educ 1990; 16: $117-122$.

6 Ferraro LA, Price JH, Desmond SM, Roberts SM: Development of a diabetes locus of control scale. Psychol Rep 1987; 61: 763-770.

7 Rosenstock IM: Understanding and enhancing patient compliance with diabetic regimens. Diabetes Care 1985; 8: $610-616$.
8 Given CW, Given BA, Gallin RS, Condon JW: Development of a scales to measure the beliefs of diabetic patients. Res Nurs Health 1983; 6: 127-141.

9 Becker MH, Janz NK: The health belief model applied to understanding diabetes regimen compliance. Diabetes Educ 1985; 11: 41-47.

10 Hurley AC: The health belief model: evaluation of a diabetes scale. Diabetes Educ 1990; 16: 44-48.

11 deWeerdt I, Visser AP, VanderVeen EA: Attitude behaviour theories and diabetes education programmes. Patient Educ Couns 1989; 14: 3-19.

12 Ajzen I, Fishbein M: Understanding Attitudes and Predicting Social Behavior. Englewood Cliffs, NJ: Prentice Hall, 1980.

13 Davis WK, Hess GE, Hiss RG: Classification of diabetes type from clinical data. Diabetes 1988; 37:260A.

14 Cattell R: The scree test for the number of factors. Multivar Behav Res 1966; 1: 140-161.

Correspondence to:

R.M. Anderson

Michigan Diabetes Research and Training Center

Towsley Center for Continuing Medical Education

Room G-1201, Box 0201

Ann Arbor, Michigan 48109-0201, USA 